Research Paper

\title{
Tumor Spheroids as an In Vitro Model for Determining the Therapeutic Response to Proton Beam Radiother- apy and Thermally Sensitive Nanocarriers
}

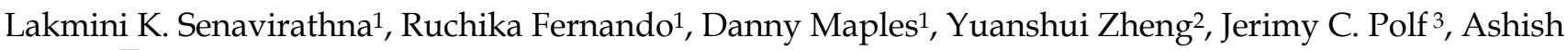 \\ Ranjan $^{1 凶}$ \\ 1. Laboratory of Nanomedicine and Targeted Therapy, Department of Physiological Sciences, Center for Veterinary Health Sciences Ok- \\ lahoma State University \\ 2. Procure Proton Therapy Center, Oklahoma City \\ 3. Department of Physics, College of Arts and Sciences, Oklahoma State University
}

$\square$ Corresponding author: Dr. Ashish Ranjan, Ph.D. 169 McElroy Hall, Physiological Sciences, Center for Veterinary Health Sciences, Stillwater, Oklahoma. Phone: 405-744-6292; Fax: 405-744-8263; Email: ashish.ranjan@okstate.edu

( ) Ivyspring International Publisher. This is an open-access article distributed under the terms of the Creative Commons License (http://creativecommons.org/ licenses/by-nc-nd/3.0/). Reproduction is permitted for personal, noncommercial use, provided that the article is in whole, unmodified, and properly cited.

Received: 2013.04.01; Accepted: 2013.06.07; Published: 2013.08.21

\begin{abstract}
Multicellular Tumor Spheroids (MCTS) strongly resemble tumor tissues, which makes them useful tools for radiation biology studies and screening of various chemotherapeutics. The goal of this pilot study was to use MCTS as an in vitro model to determine the response of cells to low temperature-sensitive liposomes (LTSLs) encapsulating doxorubicin (Dox) and proton beam radiotherapy (PBRT). Prior to treatment, MCTS were characterized for morphology and LTSLs were characterized for size, encapsulation efficiency, and ability to thermally release Dox (a model anticancer agent). Two groups of MCTS were treated with LTSL in combination with mild hyperthermia $\left(40-42{ }^{\circ} \mathrm{C}\right)$ or PBRT alone in the presence of appropriate controls. Cytotoxic response was assessed after 48-72 h using an acid phosphatase assay. At $72 \mathrm{~h}$, LTSL in combination with heat significantly reduced the viability of MCTS $(15-30 \%)$ compared to the control $(P<0.05)$. A similar cytotoxic response was observed with PBRT treatment. The data suggest that like a monolayer cell culture, MCTS can be used to determine cytotoxic outcomes of thermal and proton therapy.
\end{abstract}

Key words: Tumor spheroids, Liposomes, Proton beam radiotherapy, Drug delivery

\section{Introduction}

Monolayer cell cultures are extensively employed in drug screening and radiotherapy studies. However, their ability to reliably predict in vivo therapeutic responses is limited because they do not resemble the tumor microenvironment [1]. Three-dimensional (3D) multicellular tumor spheroid (MCTS) models have been proposed to address this shortcoming [2]. MCTS are spherical symmetric cell aggregates that can simulate the in vivo conditions and thus serve as in vitro models for hypoxia, $\mathrm{pH}$ gradient, gene expression, and drug screening studies [3]. Despite increased range of use, the application of MCTS in mild hyperthermia $\left(40-42{ }^{\circ} \mathrm{C}\right)$ and proton beam radiation (PBRT)-based studies to understand radiobiological outcomes is limited. The objective of this study was to investigate the ability of MCTS to reliably determine the therapeutic response of cells to PBRT and low temperature-sensitive liposomes (LTSLs).

The unique properties of MCTS allow high 
throughput analysis of radiobiological phenomena (Fig. 1). The core of MCTS is similar to the avascular hypoxic regions of tumors, and relies on diffusion-mediated particle transport [4-6]. In our previous in vivo studies, we achieved improved intratumoral delivery and distribution of systemically administered doxorubicin (Dox) within both the tumor periphery and core by inducing rapid intravascular doxorubicin release from LTSLs [7]. These LTSLs have lysolipids incorporated into the liposomal membrane, which allows for rapid release of their contents in response to mild hyperthermia $\left(40-42^{\circ} \mathrm{C}\right)$. A variety of methodologies (e.g., water bath hyperthermia, laser, high intensity focused ultrasound, and microwave) can be used to achieve local mild hyperthermia in tumor and impact drug delivery systems. Howev- er, proof of concept studies in animal models are generally costly, thus identifying less-expensive MCTS-based in vitro tumor alternatives that are reliable for initial high throughput analysis is essential for future cost containment. Similarly, PBRT is increasingly preferred over traditional $\mathrm{X}$-ray-based radiotherapy due to its relatively better side effect profiles. The goal of PBRT, as with all radiation therapy, is to deliver a lethal dose of radiation to a tumor while sparing surrounding healthy tissues $[8,9]$. Like LTSLs, current methods to determine PBRT effects rely on animal models or clinical trials. By choosing MCTS that have tumor-like properties, we aim to standardize methods to better reflect in vivo outcomes and improve understanding of treatment response to various dose combinations.
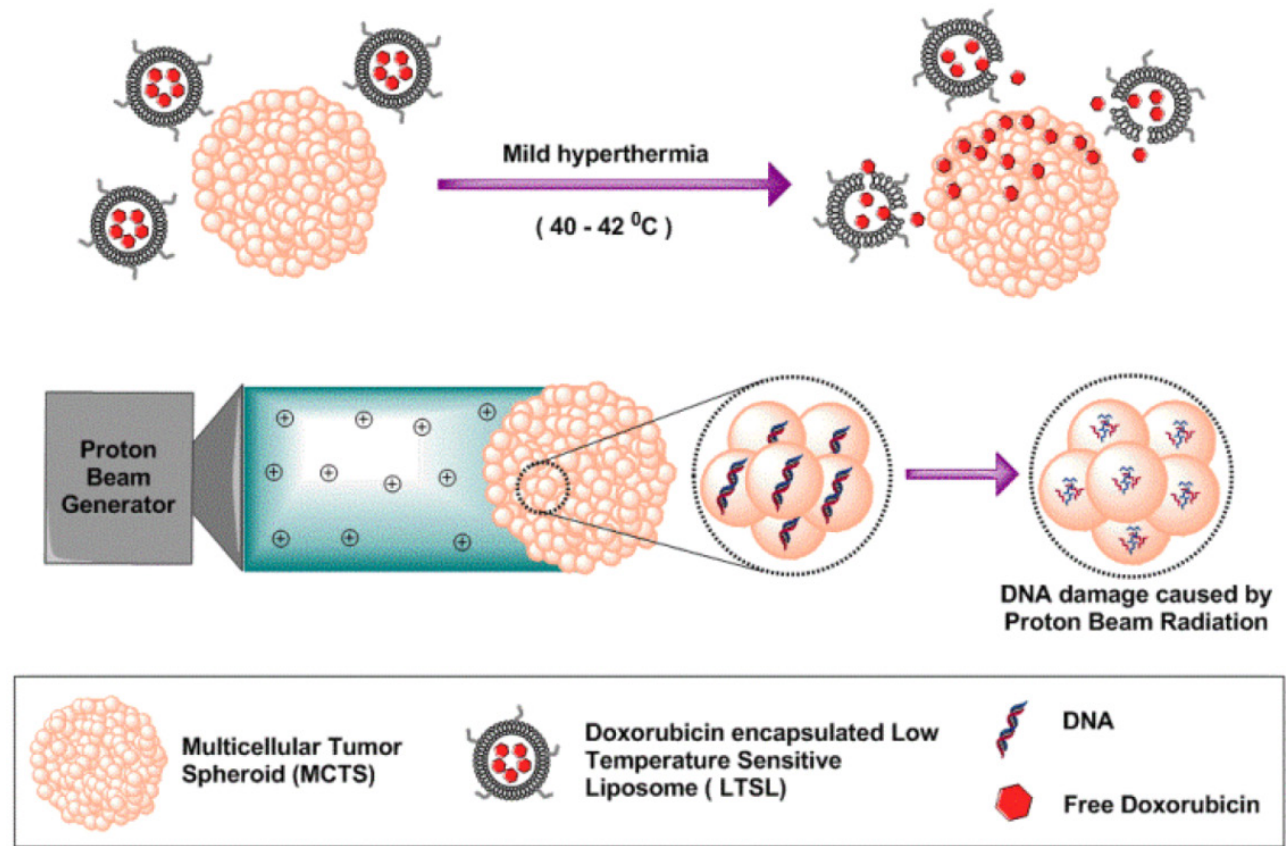

Figure I. Schematic illustration of tumor spheroids as an in vitro model for determining therapeutic efficacy against proton beam radiotherapy and doxorubicin encapsulated low temperature sensitive liposomes.

\section{Materials and Method}

\section{Synthesis of liposomes}

LTSLs were prepared by hydration of a lipid film followed by extrusion as described previously [10]. Briefly, a mixture of three phospholipids (monostearoyl-2-hydroxy-sn-glycero-3-phosphocholine, 1,2dipalmitoylsn-glycero-3-phosphocholine, and 1,2-distearoyl-sn-glycero-3-phosphoethanolamine- $\mathrm{N}$-[metho xy (polyethylene glycol)2000]) were dissolved in chloroform at a molar ratio of 85.3:9.7:5.0. The solvent was evaporated and the resulting lipid film was hydrated with citrate buffer ( $\mathrm{pH} 4.0$ ) and extruded to yield a final lipid concentration of $50 \mathrm{mg} \mathrm{mL}^{-1}$. Encapsulation of Dox into the LTSLs was carried out using a $\mathrm{pH}$ gradient loading protocol as described by Mayer et al. [11]. Prior to in vitro studies, the size of LTSLs was characterized by dynamic light scattering (DLS) using a ZetaPALS Phase Analysis Light Scattering instrument (Brookhaven Instruments Corporation, NY, USA). Dox release as a function of temperature $\left(25,37-42{ }^{\circ} \mathrm{C}\right)$ was assessed spectrophotometrically.

\section{Formation and characterization of MCTS}

All studies were performed using adenocarcinomic human alveolar basal epithelial (A549) cells as 
the model MCTS. Uniform sized MCTS were prepared using a liquid overlay technique by coating 96-well plates with $1 \%$ agarose $(\mathrm{w} / \mathrm{v})$ in phosphate buffered saline (PBS). A549 cells were seeded at 1-2 $\times$ $10^{4}$ cells per well in $200 \mu \mathrm{L}$ of RPMI 1640 supplemented with $10 \%$ fetal bovine serum and $1 \%$ penicillin/streptomycin. Cells were incubated at $37^{\circ} \mathrm{C}$ with $5 \% \mathrm{CO}_{2}$ for 2-3 days. For cell morphology characterization using scanning electron microscopy (SEM), MCTS were collected, fixed with $10 \%$ formaldehyde, washed with sodium cacodylate buffer, incubated for $1 \mathrm{~h}$ in $1 \%$ osmium tetraoxide in cacodylate buffer, serially dehydrated in increasing concentrations of ethanol $(50,7090,95$, and $100 \%)$, dried in hexamethyldisilazane, coated with gold-palladium, and viewed under the SEM (FEI Quanta 600 field emission gun ESEM, Oregan, USA).

\section{Mild hyperthermia treatment of MCTs in combination with LTSLs}

Stock solutions of LTSLs and free Dox were diluted in cell culture medium and added to MCTS at a final concentration of $30 \mu \mathrm{M}$ Dox. Drug delivery was achieved by heating the medium containing the MCTS $\left(\mathrm{n}=8\right.$ ) to $40-42{ }^{\circ} \mathrm{C}$ for $30 \mathrm{~min}$ by placing 96-well plates in a $\mathrm{CO}_{2}$ incubator. MCTS then were further incubated for $3.5 \mathrm{~h}$ at $37^{\circ} \mathrm{C}$. Free Dox, LTSLs, and untreated cells were similarly incubated for $4 \mathrm{~h}$ at $37^{\circ} \mathrm{C}$ to serve as controls. Following this treatment, MCTS were washed with PBS and re-incubated at 37 ${ }^{\circ} \mathrm{C}$ with $5 \% \mathrm{CO}_{2}$ for $72 \mathrm{~h}$ in fresh culture medium. Finally, cytotoxicity was assessed using the acid phosphatase (APH) cytotoxicity assay[12].

\section{PBRT treatment of MCTS}

PBRT treatment was conducted as follows. MCTS ( $\mathrm{n}=5,3$ replicates) in 24-well plates were placed at the center of a tissue equivalent plastic phantom to mimic the irradiation environment present inside a patient during treatment. Plates containing MCTS were irradiated with a proton dose of 0 (control), 3, or $6 \mathrm{~Gy}$, which corresponds to $0,3.3$, or 6.6 cobalt gray equivalent. The proton beam had energy of $150 \mathrm{MeV}$ and an irradiation field size of $10 \times 10 \mathrm{~cm}^{2}$. The fraction of cells in each culture that survived for each delivered dose level was determined using the APH cytotoxicity assay after $48 \mathrm{~h}$.

\section{Cytotoxicity analysis}

Cytotoxicity was assessed using the APH assay. First, an APH standard curve was established to show the linearity between the number of MCTS (2-8 spheroids) and $405 \mathrm{~nm}$ absorbance. Briefly, MCTS were collected, washed with PBS, and incubated for
90 min in the APH assay mixture [ APH buffer $(0.1 \mathrm{M}$ sodium acetate and $0.1 \%(\mathrm{v} / \mathrm{v})$ triton $-\mathrm{X}-100)$ and PBS in 3.0: 2.5 ratio $2 \mathrm{mg} /, \mathrm{L}$ of p-nitrophenyl phosphate] in an incubator at $37^{\circ} \mathrm{C}$. After incubation, $1 \mathrm{~N} \mathrm{NaOH}$ was added to the supernatant and absorbance was measured at $405 \mathrm{~nm}$. A similar protocol was used to determine the cytotoxicity in the various treatment groups.

\section{Statistical analysis}

Treatment groups were compared for differences in mean absorbance using ANOVA followed by Tukey's multiple comparisons using GraphPad Prism 6 (GraphPad Software Inc.). A p-value less than 0.05 indicated statistical significance.

\section{Results}

\section{LTSL characterization}

The hydrodynamic diameter of LTSLs measured by DLS was $106.4 \pm 0.5 \mathrm{~nm}$. Active loading of Dox by transmembrane $\mathrm{pH}$ gradient yielded an encapsulation efficiency of $>95 \%$ for Dox-LTSLs. Dox release at 37 ${ }^{\circ} \mathrm{C}$ was minimal compared to that at room temperature. However, nearly complete release of Dox from the LTSLs was observed at $42{ }^{\circ} \mathrm{C}$ (Fig.2) [10].

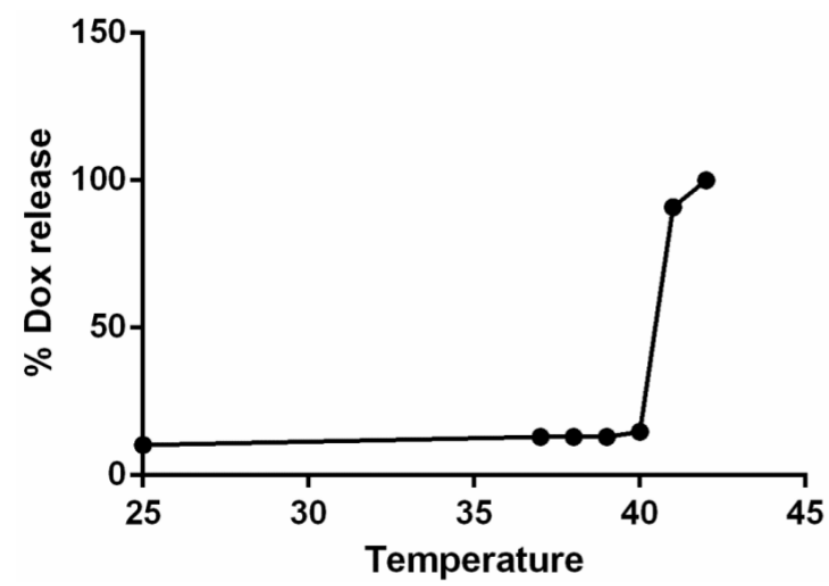

Figure 2: Dox-LTSL absorbance as a function of temperature. Nearly $100 \%$ of the drug was released at $\sim 42^{\circ} \mathrm{C}$.

\section{Generation of MCTS and their morphology}

The use of $1 \%$ agarose for coating 96-well plates provided a non-adherent surface suitable for growing MCTS that mimic in vivo tumor characteristics. At 2-3 $\mathrm{d}$ of incubation, mature spheroids around $500 \mu \mathrm{m}$ in diameter were observed. At higher magnification under SEM, compactly packed cells in the form of 3D spherical aggregates were observed with no loss of integrity due to sample preparation (Fig. 3). 


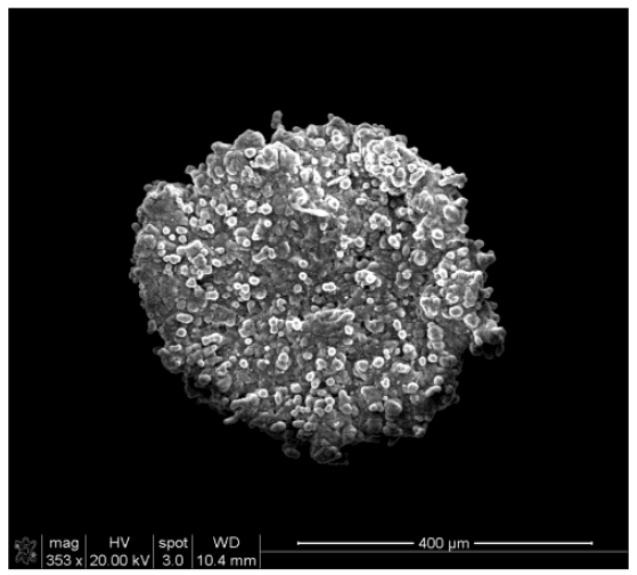

Figure 3: Scanning electron microscopic images of MCTS with a diameter of $\sim 400 \mu \mathrm{m}$.

\section{Therapeutic efficacy of LTSLs in combination with mild hyperthermia}

The APH standard curve showed a linear relationship $\left(\mathrm{R}^{2}=0.9969\right)$ between the number of MCTS and $405 \mathrm{~nm}$ absorbance (Fig. 4). As the number of MCTS increased, a proportional increase in absorbance at $405 \mathrm{~nm}$ was observed. In the drug response study (Fig. 5), at $37{ }^{\circ} \mathrm{C}$ free Dox- treated MCTS showed significantly lower (20-30\%) viability from that in the other treatment groups (LTSL, untreated control). Conversely, at $42{ }^{\circ} \mathrm{C}$, both free dox and mild hyperthermia in combination with LTSLs resulted in significantly reduced (20-30\%) viability compared to treatments without heat. Hyperthermia alone did not affect cell viability.

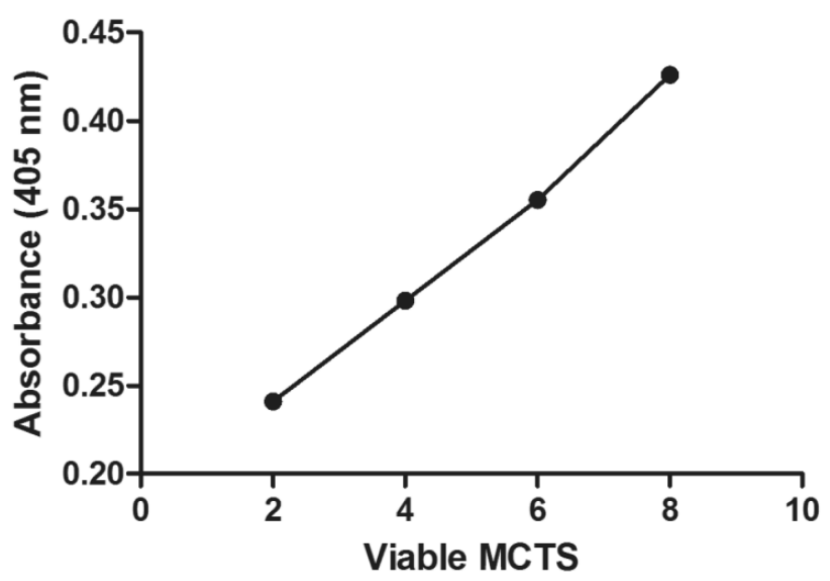

Figure 4: Viability of MCTs determined using the acid phosphatase assay. Absorbance was positively correlated with the number of MCTS.

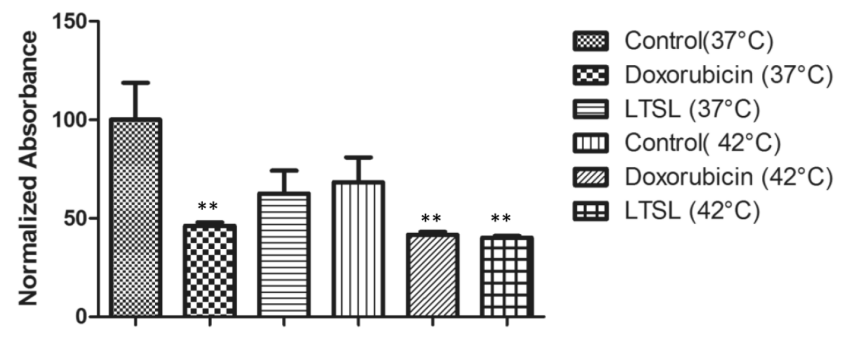

Figure 5: Viability of MCTS treated with LTSLs in combination with mild hyperthermia $(P<0.05$; significantly different than the control).

\section{Therapeutic efficacy of PBRT}

PBRT treatment of MCTS resulted in a reproducible dose-dependent reduction in cell viability (Fig. 6). A $15-20 \%$ reduction in viability compared to untreated controls was observed at $6 \mathrm{~Gy}$.

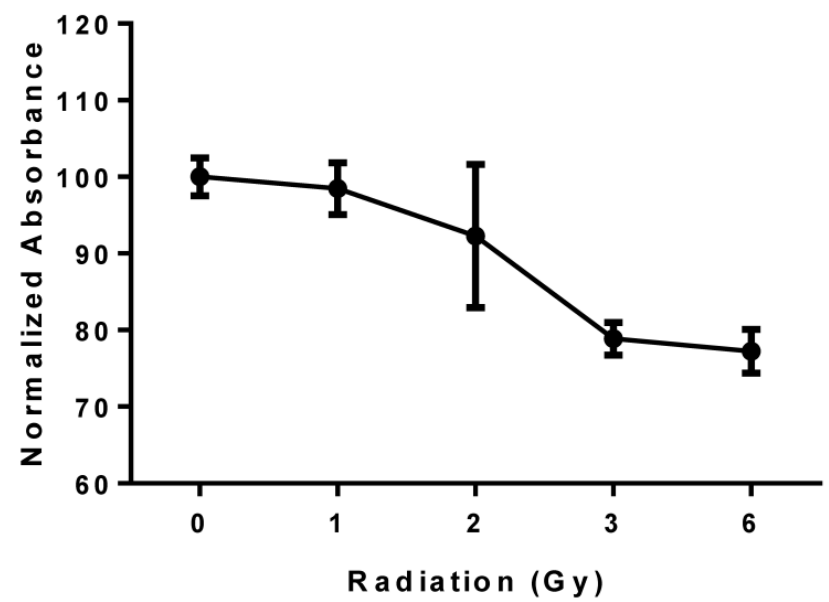

Figure 6: Viability of MCTS treated with PBRT; the plot shows a reproducible dose-dependent decrease in cell viability.

\section{Discussion}

Chemo- and radiotherapy of tumors are established treatment modalities for malignancies in which locoregional control is necessary [13]. Currently, a significant amount of active research is aimed at improving the therapeutic response of cells using novel LTSLs and radiation treatments that can achieve targeted killing of tumor cells. The MCTS method reported herein was used to test the ability of mild hyperthermia LTSL drug delivery and PBRT to enable high throughput screening of candidate nanocarriers for potential clinical translation. Cytotoxicity was evaluated using the APH assay [12]. This assay measures the ability of the enzyme $\mathrm{APH}$, which is present in viable cells, to catalyze the conversion of the chromogenic substrate p-nitrophenyl phosphate into p-nitrophenol. In the presence of an alkaline solution, this process produces the yellow-colored 
product p-nitrophenolate, which absorbs optimally at $405 \mathrm{~nm}$. Thus, the presence of this enzyme in viable cells is positively correlated with the product formed, and the absorbance of the final product is positively correlated with the viability of the MCTS. We found no statistical difference in the enzyme production between the control and heated cells (Fig. 3), which is important because hyperthermia in vitro can be difficult to control. However, significantly lower enzyme production was detected in MCTS that were treated with mild hyperthermia in combination with LTSLs. This finding suggests that the treatment triggered sufficient release of Dox from LTSLs in the targeted site at $42{ }^{\circ} \mathrm{C}$, resulting in cell death. Furthermore, the cytotoxic responses were similar between LTSLs that were initially heated to induce Dox release and those that were heated in the incubators (data not shown), indicating the reliability of this methodology.

Similarly, quantitative measurement of absorbance in MCTS treated with PBRT revealed a progressive decrease in absorbance in each treated group but a sustained increase in enzyme activity over $72 \mathrm{~h}$ in the untreated MCTS (Fig. 4). These findings are consistent with results of the traditional colonogenic assay method that measures the fraction of cells surviving for each delivered dose by incubating the cells at $37^{\circ} \mathrm{C}$ for 2 weeks to allow surviving cells to form colonies, which can be ultimately stained and counted [10].

In conclusion, the results presented herein suggest that use of MCTS can reduce the time required to estimate the success of PBRT or thermal therapy and that MCTS respond in a manner similar to that of monolayer cell culture models. In future studies, we will use this technique in theranostics assays as a versatile and simple strategy to longitudinally study nanoparticle properties in an in vitro setting.

\section{Acknowledgement}

This work was supported by a multidisciplinary team grant from the Center for Veterinary Health Sciences and the College of Arts and Sciences, Oklahoma State University, and a collaborative research agreement with ProCure Proton Therapy Center, Oklahoma City.

\section{Competing Interests}

The authors have declared that no competing interest exists.

\section{References}

1. Ho WY, Yeap SK, Ho CL, Rahim RA, Alitheen NB. Development of multicellular tumor spheroid (MCTS) culture from breast cancer cell and a high throughput screening method using the MTT assay. PloS one. 2012; 7: e44640.
2. Perche F, Patel NR, Torchilin VP. Accumulation and toxicity of antibody-targeted doxorubicin-loaded PEG-PE micelles in ovarian cancer cell spheroid model. Journal of controlled release : official journal of the Controlled Release Society. 2012; 164: 95-102.

3. Huo S, Ma H, Huang K, Liu J, Wei T, Jin S, et al. Superior penetration and retention behavior of $50 \mathrm{~nm}$ gold nanoparticles in tumors. Cancer research. 2013; 73: 319-30.

4. Cho K, Shin HW, Kim YI, Cho CH, Chun YS, Kim TY, et al. Mad1 mediates hypoxia-induced doxorubicin resistance in colon cancer cells by inhibiting mitochondrial function. Free radical biology \& medicine. 2013; 60: 201-10.

5. Grainger SJ, Serna JV, Sunny S, Zhou Y, Deng CX, El-Sayed ME. Pulsed ultrasound enhances nanoparticle penetration into breast cancer spheroids. Molecular pharmaceutics. 2010; 7: 2006-19.

6. Jin S, Ma X, Ma H, Zheng $K$, Liu J, Hou $S$, et al. Surface chemistry-mediated penetration and gold nanorod thermotherapy in multicellular tumor spheroids. Nanoscale. 2013; 5: 143-6.

7. Ranjan A, Jacobs GC, Woods DL, Negussie AH, Partanen A, Yarmolenko PS, et al. Image-guided drug delivery with magnetic resonance guided high intensity focused ultrasound and temperature sensitive liposomes in a rabbit Vx2 tumor model. Journal of controlled release. 2012; 158: 487-94.

8. Polf JC, Bronk LF, Driessen WH, Arap W, Pasqualini R, Gillin M. Enhanced relative biological effectiveness of proton radiotherapy in tumor cells with internalized gold nanoparticles. Applied physics letters. 2011; 98: 193702.

9. Kim JK, Seo SJ, Kim KH, Kim TJ, Chung MH, Kim KR, et al. Therapeutic application of metallic nanoparticles combined with particle-induced x-ray emission effect. Nanotechnology. 2010; 21: 425102.

10. Negussie AH, Yarmolenko PS, Partanen A, Ranjan A, Jacobs G, Woods $\mathrm{D}$, et al. Formulation and characterisation of magnetic resonance imageable thermally sensitive liposomes for use with magnetic resonance-guided high intensity focused ultrasound. Int J Hyperther. 2011; 27: 140-55.

11. Mayer LD, Bally MB, Cullis PR. Uptake of adriamycin into large unilamellar vesicles in response to a $\mathrm{pH}$ gradient. Biochimica et biophysica acta. 1986; 857: 123-6.

12. Friedrich J, Eder W, Castaneda J, Doss M, Huber E, Ebner R, et al. A reliable tool to determine cell viability in complex 3-d culture: the acid phosphatase assay. Journal of biomolecular screening. 2007; 12: 925-37.

13. Seiwert TY, Salama JK, Vokes EE. The concurrent chemoradiation paradigm-general principles. Nature clinical practice Oncology. 2007; 4: 86-100. 